\title{
Multiple-Scale Interface Fracture Analysis for Thin Film/Substrate System Under Temperature Mismatch
}

\author{
M.Z. Li, H.S. Ma, L.H. Liang, and Y.G. Wei
}

\begin{abstract}
Based on the Wei-Xu model (Int J Plast, 21:2123, 2005), a multiplescale interface fracture analysis for the thin film/substrate system under temperature mismatch is carried out. The thin film delamination process at the macroscopic scale is analyzed by using the strain gradient plasticity theory. The plastic shielding effects on the interface crack propagation are investigated for both the elastic film/plastic substrate and the plastic film/elastic substrate systems. On the other hand, by presenting a bridging model (equivalent interface $\mathrm{K}$ '-field) and by using the RiceThomson discrete dislocation model, the microscopic fracture process of the thin film/substrate system is analyzed, and the dislocation shielding effects on interface cracking at the microscale are investigated. The results show that the variation of interface fracture energy has an inverse size effect with respect to the dislocationfree zone size.
\end{abstract}

Keywords Multiple-scale fracture • Thin film • Temperature mismatch • Fracture energy $\bullet$ Discrete dislocation

\section{Introduction}

The interfacial fracture behavior of a structured material, such as a thin film/substrate system, is governed by processes occurring over a diverse range of length scales. This is a multiple-scale problem. Clearly, the concerned research topics belong to the science frontier, and have been focusing great attention for several decades. Until recent years, research on the interface fracture behavior of the thin film/substrate systems is still hot topic. The multiple-scale approaches

M.Z. Li • H.S. Ma • L.H. Liang • Y.G. Wei (四)

LNM, Institute of Mechanics, Chinese Academy of Sciences, Beijing 100190, China e-mail: limz@Lnm.imech.ac.cn; mahs@Lnm.imech.ac.cn; lianglh@Lnm.imech.ac.cn; ywei@Lnm.imech.ac.cn 
toward materials modeling have led to a wealth of understanding of materials mechanical behavior within each domain of model's applicability. However, it is still of central importance to address the linkage between the models at the different length scales in order to develop mechanism based modeling of mechanical behavior of materials. Several research activities can be connected to advance this objective. Based on first principle methods, interface adhesion behavior has been assessed and investigated for a series of metal/ceramic interfaces [1-4]. Through combinations of first principle analysis with continuum mechanics analysis, the interface toughness has been studied by using a multiple scale method $[2,5]$. Coating layer delamination influenced by defects (small cracks) under conditions of high thermal flux associated with a through-thickness temperature gradient has been studied in detail $[6,7]$. Another multiple-scale model combining continuum mechanics analysis at the macroscopic scale with discrete dislocation analysis at the microscopic scale has been presented [8], in which, in the continuum mechanics model, the strain gradient plasticity theory is adopted in order to describe the high microscopic strength; otherwise the stress at the microscopic scale never attains the bond rupture level. The multiple-scale characterization can provide the proper parameters for experimental measurements $[9,10]$.

In the present research, we generalize our previous multiple-scale model [8] that is built upon the elastic-plastic and strain gradient models for plastic deformation at the macroscopic scale and the discrete dislocation for dislocation shielding at the submicron scale to an analysis for thin film delamination along substrates due to temperature mismatch for the bi-materials. In our model, we attempt to establish the linkage between these two models to address the large disparity between the relevant length-scales involved in the delamination processes at the interfacial crack tip. For the macroscopic analysis model, we use a two-parameter criterion to characterize the fracture process, as usually employed for elastic-plastic fracture analyses [11-14]. The two-parameter criterion adopted here is an elastic-core model (or dislocation-free zone model, or so called SSV model $[15,16]$ ). Metal will be treated as a strain gradient plasticity material $[17,18]$, while ceramic is treated as an elastic material. There is an extensive research literature on the discrete dislocation model [19-22]. We investigate the dislocation shielding effect on the thin film delamination by considering an elastic mixed mode $\mathrm{K}$ '-field applied on the outer boundary. The K'-field and its radius is related to the macroscopic crack-tip fracture toughness through energy equivalence.

\section{Model Descriptions}

A linkage model for the thin film delamination is presented, as shown in Fig. 1. The entire description of thin film delamination should consist of both the macroscopic and microscopic interface fracture processes. The interface fracture characteristics can be described by using the continuum model and the discrete dislocation model, respectively, as sketched in Fig. 1a-d. Figure 1a and b show macroscopic fracture 

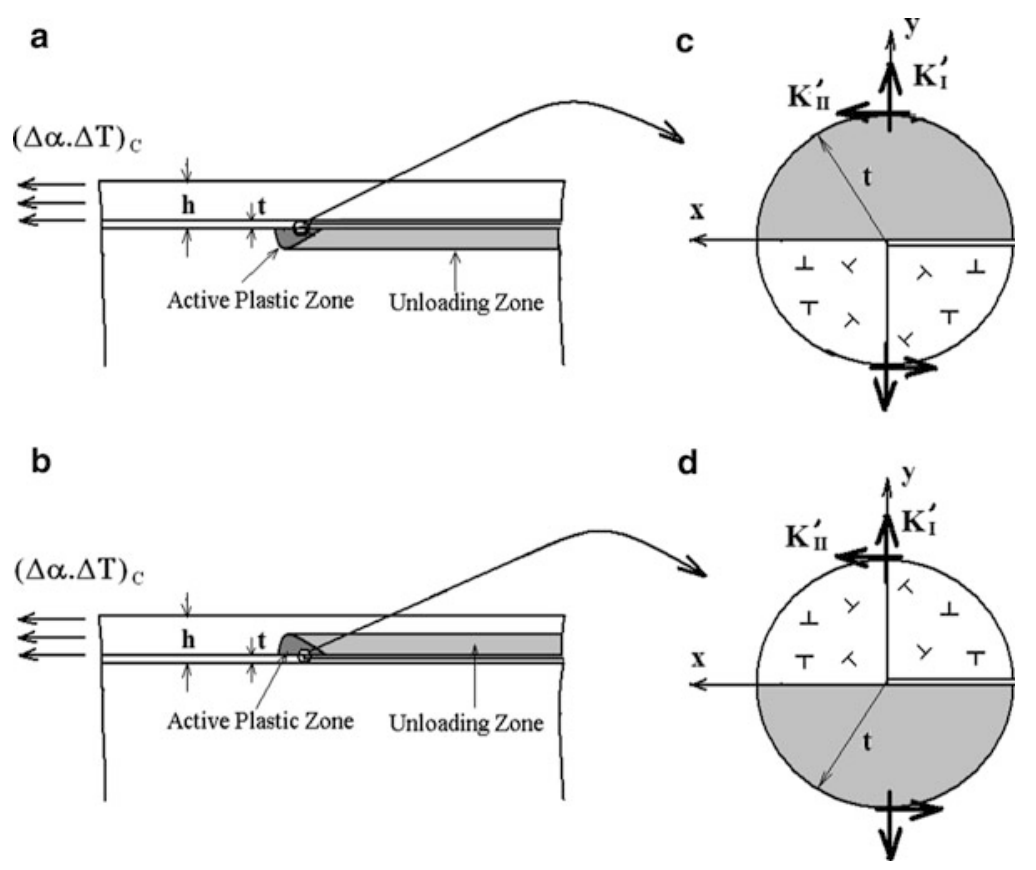

Fig. 1 Multi-scale model for thin film delemination due to temperature mismatch

models for the ceramic film/metal substrate and the metal film/ceramic substrate, respectively. The interface cracks propagate steadily under temperature mismatch. Figure $1 \mathrm{c}$ and d show micro-scale fracture models. Discrete dislocations emit and move as the crack propagates. Macroscopic fracture models corresponding to the scale level which is larger than a micron or a sub-micron can be characterized by using the conventional continuum model, i.e. the conventional elastic-plastic theory or the strain gradient theory. At the macroscopic scale, with increasing loading there exist three regions around the interface crack tip: the elastic zone far away from the tip, the strain gradient dominated zone very near the crack tip and the plastic zone between the elastic zone and the strain gradient zone. In order to describe the multiple-scale problem effectively, an elastic core model with thickness $t$ [15] is adopted here, as shown in Fig. 1a and b. This model supposes that near the growing crack tip and along the crack surface there exists a dislocation-free strip with thickness $t$ and infinite length.

Under the condition of steady-state crack growth, the thickness of the elastic layer, $t$, can be taken as a model parameter [23, 24]. Within a much smaller scale (smaller than a micron or sub-micron), the interface fracture behavior is characterized by the discrete dislocation theory, as shown in Fig. 1c and d. With load increase, dislocations nucleate and emit from the crack tip, and exist (remain in equilibrium) within the region. Several problems need to be addressed: What is the number of discrete dislocations in equilibrium or in limit equilibrium? What 
is the effective size of the discrete dislocation region, $t$ ? In addition, what are the linkage conditions for the macroscale and the microscale, i.e., the outer boundary conditions of the elastic core? According to energy equivalence, the results based on the macroscopic analysis will be taken as the outer boundary conditions of the microscopic problem with an equivalent $\mathrm{K}^{\prime}$-field exerted, which is based on the observation that the crack tip field predicted by the strain gradient theory has about $(-1 / 2)$ singularity [8]. The key point is to determine the intersection radius between the microscopic problem and macroscopic problem.

\section{Thin Film Delamination Analysis by Using the Continuum Model}

Specifically, we shall focus our attention on thin film delamination under a temperature mismatch. The corresponding model is shown in Fig. 1a and b. Using the SSV model and the compressible strain gradient plasticity flow theory [17], through dimensional analysis, the relations of the normalized total energy release rate with the material parameters and the model parameter can be written as

$$
\frac{G_{c r i t}}{G_{0}^{\prime}}=f\left(\frac{E}{\sigma_{Y}}, \frac{E_{f}}{E_{s}}, v_{f}, v_{s}, n, \frac{h}{R_{0}}, \frac{l}{R_{0}}, \frac{R_{0}}{t}\right)
$$

where the length parameter $l$ describes the strain gradient effect for metal, $E$ is Young's modulus for metal, $t$ is the elastic core size to be determined, $n$ is the strain hardening exponent, $E_{f}$ and $E_{s}$ are Young's modulus for film and substrate, respectively. A length parameter $R_{0}$ is defined as follows

$$
R_{0}=\frac{E G_{0}^{\prime}}{3 \pi\left(1-v^{2}\right) \sigma_{Y}^{2}}
$$

which is the plastic zone size in small scale yielding. $G_{0}^{\prime}$ is the macroscopic fracture toughness at the tip. $E$ is the Young's modulus and $v$ is Poisson's ratio for metal.

For thin film delamination due to a temperature mismatch, the total energy release rate can be expressed as

$$
G_{c r i t}=\frac{1}{2}\left(1-v^{2}\right) E_{f}(\Delta \alpha \cdot \Delta T)_{c}^{2} h
$$

where $\Delta \alpha$ and $\Delta T$ are the mismatches of the thermal expansion coefficient and the temperature between film and substrate, respectively. Through finite element numerical simulation, the parameter relation (1) can be obtained and is plotted in Fig. 2 for several parameter values and for the case of the ceramic film/metal substrate. From Fig. 3, variation of the normalized energy release rate is very sensitive to the elastic core size and the length parameter $R_{0}$. For a typical metal, 
Fig. 2 Relations of the normalized energy release rate vs. elastic core size based continuum mechanics analysis for the metal film/ceramic substrate

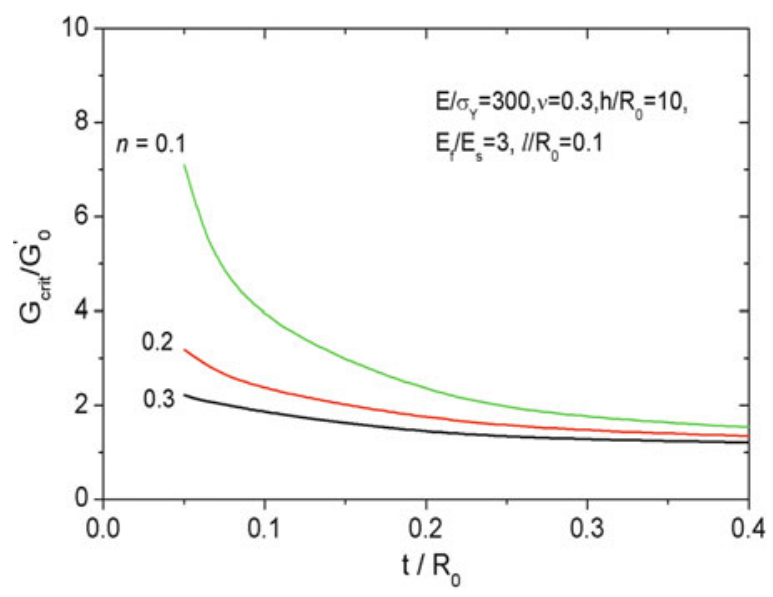

Fig. 3 Relations of the normalized energy release rate vs. elastic core size based

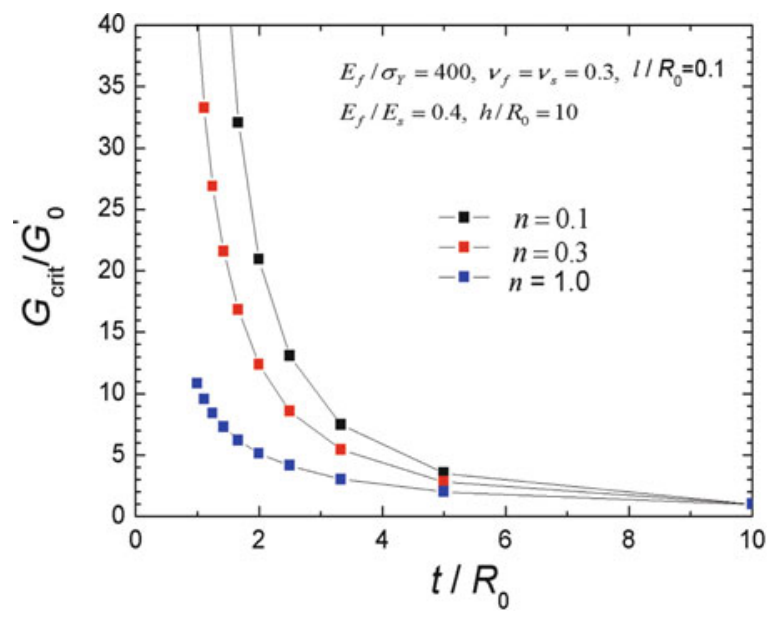

the value of $R_{0}$ is about one micron. Therefore, when the elastic core size is taken to be submicron, the normalized energy release rate is much sensitive to the value of the elastic core size.

In order to determine the outer boundary condition for the microscopic fracture analysis, the macroscopic crack tip solution is equivalently expressed into a standard interface K'-field,

$$
\begin{gathered}
G_{0}^{\prime}=\frac{1}{2}\left(1-\beta_{D}{ }^{2}\right)\left(\frac{1-v^{2}}{E}+\frac{1-v_{s}^{2}}{E_{s}}\right)\left(K_{I}^{\prime 2}+K_{I I}^{\prime 2}\right) \\
\tan \Psi=\frac{\sigma_{12}}{\sigma_{22}}=\frac{\operatorname{Im}\left[\left(K_{I}^{\prime}+i K_{I I}^{\prime}\right) L_{0}{ }^{i \varepsilon}\right]}{\operatorname{Re}\left[\left(K_{I}^{\prime}+i K_{I I}^{\prime}\right) L_{0}^{i \varepsilon}\right]}
\end{gathered}
$$


Fig. 4 Variation of mode mixity with normalized size of elastic core

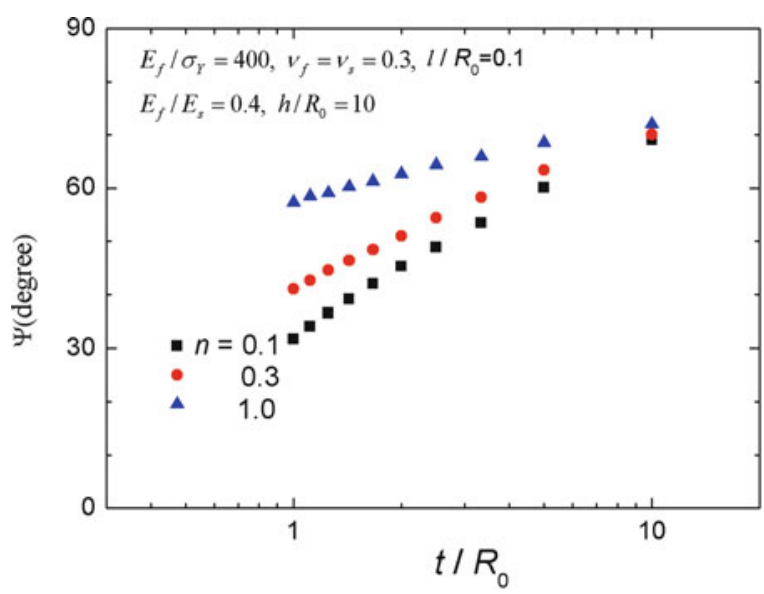

where $\Psi$ is the mode mixity, $L_{0}$ is a reference length, and

$$
\begin{gathered}
\sigma_{22}+i \sigma_{12}=\left(K_{I}^{\prime}+i K_{I I}^{\prime}\right)(2 \pi r)^{-1 / 2} r^{-\varepsilon} \\
\varepsilon=\frac{1}{2} \ln \left(\frac{1-\beta_{D}}{1+\beta_{D}}\right), \beta_{D}=\frac{1}{2} \frac{\mu\left(1-2 v_{s}\right)-\mu_{s}(1-2 v)}{\mu\left(1-v_{s}\right)+\mu_{s}(1-v)}
\end{gathered}
$$

For $E_{s}=E$ and $v_{s}=v$, then $\beta_{D}=0$ and $\varepsilon=0$, one has

$$
G_{0}^{\prime}=\frac{\left(1-v^{2}\right)\left(K_{I}^{\prime 2}+K_{I I}^{\prime 2}\right)}{E}, \tan \Psi=\frac{K_{I I}^{\prime}}{K_{I}^{\prime}}
$$

The variation of mode mixity with other parameters is calculated and the result is shown in Fig. 4. From Fig. 4, the mode mixity tends to the mode I case as $t$ decreases. Specifically, for conventional metals with weak hardening properties, mode I tends to be the dominant case. Therefore, for simplicity we consider a mode I crack field which is exerted on the outer boundary of the microscopic fracture model. Within the elastic core region, several discrete dislocations are included.

\section{Fracture Analyses Based on the Discrete Dislocation Model}

As discussed above, the macroscopic fracture process is accompanied by a microscopic fracture process. For the microscopic fracture analysis, the Rice-Thomson discrete dislocation model is adopted here. The simplified model has been presented previously, as shown in Fig. 1c and d, and as discussed above, we consider a mode I field exerted on the outer boundary. The problem is characterized by Fig. 5a and b for a stationary crack and steady-state crack growth, respectively. The continuum 

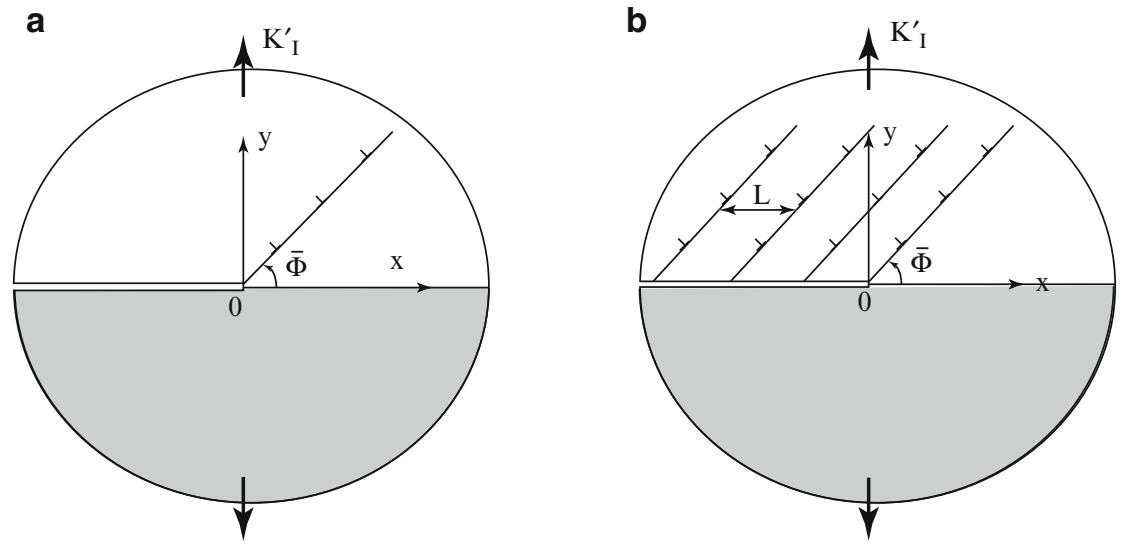

Fig. 5 Dislocation slip models for interface crack. (a) Stationary crack (b) Steady-state growing crack

solutions discussed above, a $K_{I}^{\prime}$ field, can be obtained using the first expression of Eq. (8). The key problem is how to properly select the radius of the outer boundary, $t$. The $t$ value will be determined through bridging the macroscopic with microscopic analysis results after the discrete dislocation analysis is carried out. In order to analyze how crack growth behavior at the micron scale is influenced by the discrete dislocations, as usual [19-22], a typical arrangement of discrete dislocations is considered, as shown in Fig. 5a or b. The possibility of putting as many dislocations as possible within the region $0<r<t$, will be investigated (corresponding to the limit equilibrium state for each dislocation), where $r$ is the radial polar coordinate. The arrangement of the discrete dislocations is according to the dislocation equilibrium status: $-1 \leq f_{d} / f_{d}^{c} \leq 1$, where $f_{d}$ is the dislocation force, $f_{d}^{c}=\sigma_{f} b$ is referred to as the lattice frictional resistance, $\sigma_{f}$ and $b$ are the critical shear strength along the slip plane and length of the Burgers vector, respectively. For $f_{d}=f_{d}^{c}$, a dislocation is in the limit equilibrium state. We will focus on the limit equilibrium state. It is worth noting that Lin and Thomson [20] have obtained the dislocation force formulations and the dislocation shielding effect formulations (solutions), so we can base our analysis of the interaction of the crack with discrete dislocations on the Lin and Thomson's formulations.

The macroscopic fracture solutions for steady-state crack growth are given in Sect. 3. Here we focus attention on the microscopic fracture analysis. We have considered the possibility of discrete dislocations existing along a slip plane within the submicron scale in the above sections. Let us further investigate the possibility of the pattern of dislocations being retained as the crack grows steadily, i.e. investigate whether the dislocation pattern can be kept when a new slip plane is produced near the crack tip (see Fig. 5b). Suppose that the space between slip planes is equal to $L$. A question is: what is the size of the slip plane space $L$ ? According to the experimental observation and measurement [25], $L$ is about $1 \mu \mathrm{m}$, or submicron. By calculating the dislocation forces for each dislocation or the dislocation pattern, 
Fig. 6 Variation of the toughness ratio with elastic core size based on the microscopic model

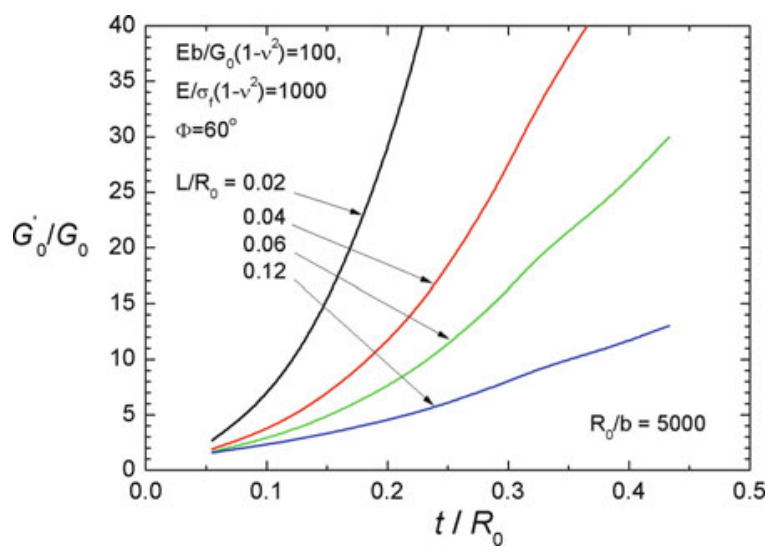

one can examine the stability of the dislocation pattern, i.e., check whether or not $\left|f_{d} / f_{d}^{c}\right| \leq 1$ is met as the crack grows. After the condition is confirmed, we obtain several results about the toughness ratio.

Under steady-state crack growth and for the microscopic fracture process, through dimensional analysis, the ratio of energy release rate to crack tip fracture energy can be expressed as the following parameter function,

$$
\frac{G_{0}^{\prime}}{G_{0}}=g\left(\frac{E}{E_{s}}, \frac{t}{b}, \frac{L}{b}, \frac{E b}{G_{0}\left(1-v^{2}\right)}, \frac{E}{\sigma_{f}\left(1-v^{2}\right)}, \bar{\Phi}\right)
$$

Figure 6 shows the variation of toughness ratio with the normalized elastic core radius, $t$, for several normalized material parameters: $E b / G_{0}\left(1-v^{2}\right), R_{0} / b, L / b$, $E / \sigma_{f}\left(1-v^{2}\right)$. This toughness ratio represents the ratio of the macroscopic crack tip toughness to the microscopic crack tip toughness.

\section{On the Determination of Elastic Core Size}

We can directly calculate how the energy release rate changes with the elastic core size $t$. From Fig. 6, the toughness ratio from microscopic theory increases with increasing elastic core size, which is the inverse of what is obtained from the macroscopic analysis (Fig. 3). Both results display an important conclusion that energy release rate has an inverse size effect with elastic core size (dislocation-free zone size). From both microscopic and macroscopic analysis results, one can obtain the total energy release rate as

$$
\frac{G_{c r i t}}{G_{0}}=\frac{G_{c r i t}}{G_{0}^{\prime}} \frac{G_{0}^{\prime}}{G_{0}}
$$


The elastic core size corresponds to the minimum value taken by the total energy release rate, or is determined by letting $\partial\left(G_{c r i t} / G_{0}\right) / \partial t=0$. From previous analysis by the author [8], the elastic core size is about $t \approx R_{0} / 6$ for a typical metal. However, for the film/substrate system, from Figs. 3 and 6, the elastic core size is around $R_{0} / 3$.

\section{Concluding Remarks}

A complete delamination process of the thin film/substrate system due to temperature mismatch has been analyzed based on the multiple-scale interface fracture model. In the multiple-scale model, the macroscopic delamination process has been described by the strain gradient plasticity theory which can realize the high strength characterization near the interface crack tip, while the microscopic fracture process has been delineated by using the Rice-Thomson discrete dislocation model, which can consider the effects of discrete dislocation shielding on crack propagation. Both macroscopic and microscopic descriptions can be bridged through energy equivalence, in which the macroscopic interface fracture energy (fracture toughness) is taken as the outer boundary condition of the microscopic fracture problem with equivalent interface $\mathrm{K}$ '-field based on the observation that crack tip field from strain gradient theory has about a $(-1 / 2)$ singularity. In the present analyses for both the ceramic thin film/metal substrate and the metal thin film/ceramic substrate, the multiple-scale interface fracture characteristics have been displayed. One of them is that the variation of interface fracture energy displays an inverse size effect with respect to the dislocation-free zone size, which may provide an explanation for the inverse Hall-Petch behavior in nanostructured materials.

Acknowledgements The work is supported by National Science Foundations of China through Grants Nos 10721202, 10932011, 90816004.

\section{References}

1. Jiang, Y., Smith, J.R.: Pt effects in gamma-Ni(Al)/alpha-Al2O3 adhesion. J. Mater. Sci. 44, 1734-1740 (2009)

2. Jiang, Y., Wei, Y.G., Smith, J.R., Hutchinson, J.W., Evans, A.G.: First principles based predictions of the toughness of a metal/oxide interface. Int. J. Mater. Res. 101, 8-15 (2010)

3. Jiang, Y., Smith, J.R., Evans, A.G.: First principles assessment of metal/oxide interface adhesion. Appl. Phys. Lett. 92, 141918 (2008)

4. Smith, J.R., Jiang, Y., Evans, A.G.: Adhesion of the gamma-Ni(Al)/alpha-Al2O3 interface: a first-principles assessment. Int. J. Mater. Res. 98, 1214-1221 (2007)

5. Wei, Y.G., Hutchinson, J.W.: Toughness of Ni/Al2O3 interfaces as dependent on micron-scale plasticity and atomistic-scale separation. Phil. Mag. 88, 3841-3859 (2008)

6. Xue, Z., Evans, A.G., Hutchinson, J.W.: Delamination susceptibility of coatings under high thermal flux. J. Appl. Mech. 76, 041008 (2009) 
7. Evans, A.G., Hutchinson, J.W.: The mechanics of coating delamination in thermal gradients. Surf. Coat. Tech. 201, 7905-7916 (2007)

8. Wei, Y.G., Xu, G.: A multiscale model for the ductile fracture of crystalline materials. Int. J. Plast. 21, 2123-2149 (2005)

9. Wei, Y.G., Zhao, H.F.: Peeling experiments of ductile thin films along ceramic substrates critical assessment of analytical models. Int. J. Solids Struct. 45, 3779-3792 (2008)

10. Hemker, K.J., Mendis, B.G., Eberl, C.: Characterizing the microstructure and mechanical behavior of a two-phase NiCoCrAlY bond coat for thermal barrier systems. Mater. Sci. Eng. A 483, 727-730 (2008)

11. Betegon, C., Hancock, J.W.: Two-parameter characterization of elastic-plastic crack-tip fields. J. Appl. Mech. 113, 104-110 (1991)

12. Tvergaard, V., Hutchinson, J.W.: The influence of plasticity on mixed mode interface toughness. J. Mech. Phys. Solids 41, 1119-1135 (1993)

13. Wei, Y., Wang, T.C.: Fracture criterion based on the higher-order asymptotic fields. Int. J. Fract. 73, 39-50 (1995)

14. Wei, Y., Wang, T.C.: Characterization of elastic-plastic fields near stationary crack tip and fracture criterion. Eng. Fract. Mech. 51, 547-553 (1995)

15. Suo, Z., Shih, C.F., Varias, A.G.: A theory for cleavage cracking in the presence of plastic flow. Acta Metall. Mater. 41, 151-1557 (1993)

16. Beltz, G.E., Rice, J.R., Shih, C.F., Xia, L.: A self-consistent model for cleavage in the presence of plastic flow. Acta Mater. 44, 3943-3954 (1996)

17. Wei, Y., Hutchinson, J.W.: Steady-state crack growth and work of fracture for solids characterized by strain gradient plasticity. J. Mech. Phys. Solids 45, 1253-1273 (1997)

18. Gao, H., Huang, Y., Nix, W.D., Hutchinson, J.W.: Mechanism-based strain gradient plasticity -I. Theory. J. Mech. Phys. Solids 47, 1239-1263 (1999)

19. Rice, J.R., Thomson, R.: Ductile versus brittle behaviour of crystals. Phil. Mag. 29, 73-97 (1974)

20. Lin, I.H., Thomson, R.: Cleavage, dislocation emission, and shielding for cracks under general loading. Acta Metall. 34, 187-206 (1986)

21. Hsia, K.J., Suo, Z., Yang, W.: Cleavage due to dislocation confinement in layered materials. J. Mech. Phys. Solids 42, 877-896 (1994)

22. $\mathrm{Xu}, \mathrm{G}$., Argon, A.S.: Critical configurations for dislocation nucleation from crack tips. Phil. Mag. A 75, 341-367 (1997)

23. Wei, Y., Hutchinson, J.W.: Nonlinear delamination mechanics for thin films. J. Mech. Phys. Solids 45, 1137-1159 (1997)

24. Wei, Y., Hutchinson, J.W.: Models of interface separation accompanied by plastic dissipation at multiple scales. Int. J. Fract. 95, 1-17 (1999)

25. Mao, S.X., Evans, A.G.: The influence of blunting on crack growth at oxide/metal interfaces. Acta Mater. 45, 4263-4270 (1997) 